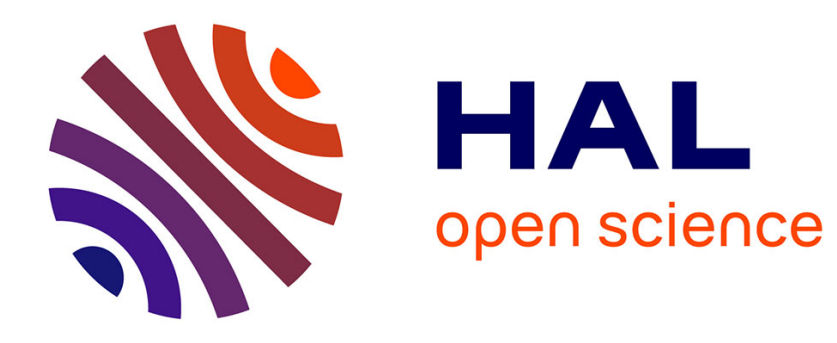

\title{
Une autre ville pour une autre vie. Henri Lefebvre et les situationnistes.
}

Philippe Simay

\section{To cite this version:}

Philippe Simay. Une autre ville pour une autre vie. Henri Lefebvre et les situationnistes.. Métropoles, 2009. hal-01949682

\section{HAL Id: hal-01949682 \\ https://hal.science/hal-01949682}

Submitted on 10 Dec 2018

HAL is a multi-disciplinary open access archive for the deposit and dissemination of scientific research documents, whether they are published or not. The documents may come from teaching and research institutions in France or abroad, or from public or private research centers.
L'archive ouverte pluridisciplinaire HAL, est destinée au dépôt et à la diffusion de documents scientifiques de niveau recherche, publiés ou non, émanant des établissements d'enseignement et de recherche français ou étrangers, des laboratoires publics ou privés. 
Numéro 4 (2008)

Aménagement urbain et transition post-keynésienne

Philippe Simay

\title{
Une autre ville pour une autre vie. Henri Lefebvre et les situationnistes
}

\author{
Avertissement \\ Le contenu de ce site relève de la législation française sur la propriété intellectuelle et est la propriété exclusive de \\ l'éditeur. \\ Les œuvres figurant sur ce site peuvent être consultées et reproduites sur un support papier ou numérique sous \\ réserve qu'elles soient strictement réservées à un usage soit personnel, soit scientifique ou pédagogique excluant \\ toute exploitation commerciale. La reproduction devra obligatoirement mentionner l'éditeur, le nom de la revue, \\ l'auteur et la référence du document. \\ Toute autre reproduction est interdite sauf accord préalable de l'éditeur, en dehors des cas prévus par la législation \\ en vigueur en France.
}

\section{revues.org}

Revues.org est un portail de revues en sciences humaines et sociales développé par le Cléo, Centre pour l'édition électronique ouverte (CNRS, EHESS, UP, UAPV).

\section{Référence électronique}

Philippe Simay, « Une autre ville pour une autre vie. Henri Lefebvre et les situationnistes », Métropoles [En ligne], 4 | 2008, mis en ligne le 18 décembre 2008. URL : http://metropoles.revues.org/2902

DOI : en cours d'attribution

Éditeur : Philippe Genestier http://metropoles.revues.org http://www.revues.org

Document accessible en ligne sur : http://metropoles.revues.org/2902

Ce document PDF a été généré par la revue.

(c) Tous droits réservés 


\section{Une autre ville pour une autre vie. Henri Lefebvre et les situationnistes}

Philippe Simay, Directeur de programme au Collège International de Philosophie, p.simay@free.fr

Résumé : L'un des traits distinctifs du mouvement situationniste est de s'être constamment préoccupé de la question urbaine. Conçue comme l'espace de production de la société du spectacle mais aussi comme un terrain de lutte et d'expérimentation, la ville a représenté pour ce mouvement estudiantin, proche d'Henri Lefebvre, le lieu d'une réinvention radicale de la vie quotidienne. Cet article revient sur la critique situationniste de l'urbanisme de l'aprèsguerre ainsi que sur les pratiques auxquelles elle a donné lieu (détournement, dérive, cartes psychogéographiques, construction de situations éphémères). Celles-ci peuvent être regardées comme la première expression d'un «droit à la ville », tel que l'entendait Lefebvre. Reste à savoir si le groupe de Guy Debord souhaitait véritablement « changer la ville pour changer la vie » ou si la ville n'était que le théâtre d'une révolution à venir.

Mots clés : Henri Lefebvre, Guy Debord, situationnistes, dérive

Summary : One of the distinctive traits of the situationist movement is its constant preoccupation with the urban issue. For this student movement close to Henri Lefebvre, the city has been thought of as a choice generic location for the production of the Society of the Spectacle, but also as a test ground for struggle and experimentation - the place where a radical reinvention of daily life can occur. This paper goes back to the situationist critique of post-war urbanism, as well as the behaviors and practices it has given birth to (misappropriation, drifts in meaning, psychogeographic maps, the construct of short-lived situations). These can be seen as the first expression of a « right to the city », in the sense Lefebvre originally meant it have. However, it is still unknown whether Guy Debord's group really wished to « change city-life to change life itself », or if to them the city was merely a theater for the enactment of a revolution to come.

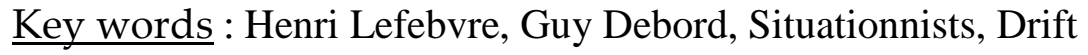

Il est peu de mouvement qui, à l'instar des situationnistes, ait placé la question urbaine au cœur de leurs pratiques artistiques et littéraires. Conçue comme l'espace de production de la société du spectacle, de la consommation et du contrôle social puis, au contraire, comme un «nouveau théâtre d'opération dans la culture », c'est-à-dire comme un terrain de lutte et 


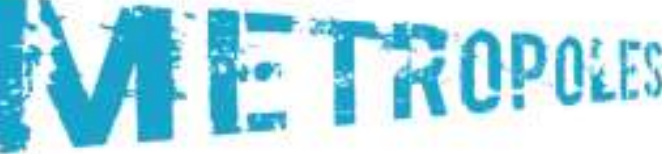

\section{$2008, n^{\circ} 4$}

d'expérimentation, la ville a représenté pour les situationnistes le lieu même d'une transformation révolutionnaire de l'existence, à travers la participation des citadins et la réintégration du poétique dans l'ordinaire. Cette préoccupation fait directement écho à celle d'Henri Lefebvre, que Debord et ses camarades rencontrent en 1960, et qui en avait posé les premiers jalons dès 1946 dans sa Critique de la vie quotidienne puis dans La somme et le reste en 1959. Entre le sociologue et les situationnistes, l'échange sera mutuellement fécond. Il sera aussi bref et tumultueux : dès 1963, les situationnistes accuseront Lefebvre de plagiat ${ }^{1}$ tandis que ce dernier se posera indûment en source d'inspiration du mouvement estudiantin ${ }^{2}$. Dans les lignes qui suivent, il ne s'agira pas de rappeler ce qui revient à chacun en dénonçant les abus d'emprunt. Cette histoire est désormais connue ${ }^{3}$. En revanche, il importe de revenir sur divers moments de la réflexion urbaine des situationnistes parce qu'elle constitue la première expression d'un droit à la ville tel que l'entendait Lefebvre et la toute première tentative pour changer la vie en changeant la ville.

\section{Une critique de l'urbanisme}

Au début du Formulaire pour un urbanisme nouveau on peut lire que «l'architecture est le plus simple moyen d'articuler le temps et l'espace, de moduler la réalité, de faire rêver. Il ne s'agit pas seulement d'articulation et de modulation plastiques, expression d'une beauté passagère. Mais d'une modulation influentielle, qui s'inscrit dans la courbe éternelle des désirs humains et des progrès dans la réalisation de ces désirs. [...] Le complexe architectural sera modifiable. Son aspect changera en partie ou totalement suivant la volonté de ses habitants. [...] Sur la base de cette civilisation mobile, l'architecture sera - au moins à ses débuts - un moyen d'expérimenter les mille façons de modifier la vie, en vue d'une synthèse

\footnotetext{
${ }^{1}$ Lefebvre reconnaîtra en effet s'être inspiré d'un texte de Guy Debord et Michèle Bernstein sur la Commune de Paris comme fête pour son propre livre, ce qui lui vaudra une sévère dénonciation dans un tract de 1963, « Aux poubelles de l'histoire », ainsi que dans les numéros 9 et 12 de l'Internationale Situationniste.

${ }^{2}$ H. Lefebvre, Le temps des méprises, Paris, Stock, 1975.

${ }^{3}$ Voir notamment G. Marelli, L'amère victoire du situationnisme, Paris, Sulliver, 1998, V. Kaufmann, Guy Debord. La révolution au service de la poésie, Paris, Fayard, 2001 et A. Jappe, L'avant-garde inacceptable : réflexions sur Guy Debord, Paris, Leo Scheer, 2004.
} 


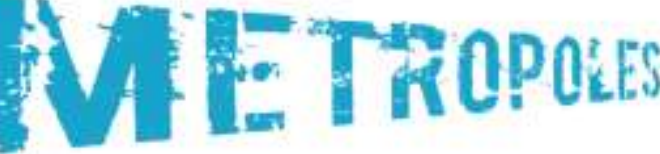

\section{$2008, n^{\circ} 4$}

qui ne peut être que légendaire ${ }^{4}$. Ce texte, rédigé en 1953 par Ivan Chtcheglov puis publié en 1958 sous le pseudonyme de Gilles Ivain dans le premier bulletin de L'Internationale situationniste, fait incontestablement figure de manifeste. On $\mathrm{y}$ trouve les traits caractéristiques de la réflexion urbaine des premiers situationnistes. Et notamment l'affirmation d'un lien consubstantiel entre la ville, la vie et le désir. A l'avenir, l'architecture et l'urbanisme devront jouer un rôle majeur dans la transformation des modes de vie, car c'est d'eux que résulte la possibilité de tout changement. L'architecture, comme pratique et comme réalisation, doit donc être reconsidérée : elle n'est pas seulement un moyen d'habitation mais avant tout un instrument de connaissance et d'action. La pratique architecturale restera-t-elle l'apanage de quelques artistes ou sera-t-elle au pouvoir de tous ? Le texte ne se prononce pas sur cette question qui constituera un différend entre certains situationnistes mais l'objectif est clair : «Contre le spectacle, la culture situationniste réalisée introduit la participation totale $»^{5}$. La pratique architecturale, entendue au sens restreint ou au sens large, doit faire de chaque citadin un acteur participant lui-même à la construction de situations, c'est-à-dire à l'élaboration de moments de vie, à la fois singuliers et collectifs, à la création d'ambiances ou de jeux d'événements, tous transitoires. De ce fait, chaque construction, pour être à l'écoute du désir et ouverte sur le possible, devra nécessairement être mobile et modulable, permettant à chacun, au gré de ses envies, des rencontres et de la force des lieux, de réinventer sa vie à travers une série indéfinie d'expérimentations.

Avant même que ne se dessinent les linéaments de cette architecture sensible, fluide et ludique, l'Internationale situationniste, ou plutôt l'organisation qui la précède, l'Internationale lettriste, a pris clairement position : l'art et la poésie ne sont plus à rechercher dans toute forme d'œuvre ni même dans les livres ; ils se situent dans la forme des villes. Il faut construire des villes bouleversantes qui ne laisseront plus de place à l'ennui. L'architecture devrait donc opérer cette transformation de la vie en œuvre d'art et libérer l'individu de l'aliénation dans laquelle le maintiennent la spécialisation du travail et la normalisation du quotidien. Or il s'en faut encore de beaucoup pour que l'architecture parvienne à changer la vie en changeant

\footnotetext{
${ }^{4}$ «Formulaire pour un urbanisme nouveau », I.S. $\mathrm{n}^{\circ} 1$, in Internationale situationniste (1958-1969), Paris, Fayard, 1997, p. 16-17.

${ }^{5}$ Internationale situationniste $\mathrm{n}^{\circ} 4$.
} 


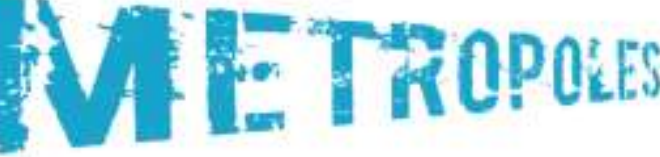

\section{$2008, n^{\circ} 4$}

simplement la ville. Pour qu'une telle entreprise réussisse, il faut préalablement détourner l'architecture des finalités utilitaires que lui impose l'urbanisme moderne et récuser les valeurs dominantes de la bourgeoisie. C'est pourquoi les premiers efforts de l'Internationale lettriste ne sont pas investis tant dans la matérialisation d'une nouvelle architecture que dans la dénonciation de la banalisation de l'existence sous l'effet de la société de consommation et de l'urbanisme intensif de l'après-guerre.

On constate, en effet, que dès le n 3 de la revue Potlatch, en juillet 1954, les jeunes lettristes accusent les effets pervers de la reconstruction. Au nom de la crise du logement, disent-ils, on voit apparaître par centaines des bâtiments dépourvus de qualité architecturale. L'article indique ironiquement que «pour éviter toute rupture d'harmonie, [les architectes urbanistes] ont mis au point quelques taudis types, dont les plans servent aux quatre coins de France. Le ciment armé est leur matériau préféré. Ce matériau se prêtant aux formes les plus souples, on ne l'emploie que pour faire des maisons carrées. [...] Dans leurs œuvres, un style se développe, qui fixe les normes de la pensée et de la civilisation occidentale du vingtième siècle et demi. C'est le style « caserne » et la maison 1950 est une boîte ${ }^{6}$.

Ce qui est évoqué ici c'est la production effrénée de ces barres de béton, toutes réalisées sur le même modèle et à partir desquelles on reconstruit de nouveaux quartiers ; ce sont ces grandsensembles dont la pauvreté formelle impose la monotonie d'un mode de vie normalisé. Pour les membres de l'Internationale lettriste, cette architecture de la banalité et de la massification ne résulte pas seulement de l'étroitesse d'esprit des constructeurs mais avant tout de principes théoriques fallacieux. Parmi ces principes, ils retiendront tous ceux qui participent d'un réductionnisme fonctionnaliste et notamment celui qui fait de la maison une «machine à habiter » où l'existence humaine est réduite à une série de besoins primaires. Ceci explique pourquoi Le Corbusier, chef de file du mouvement moderne, devient vite la cible privilégiée des lettristes : «avec Le Corbusier, les jeux et les connaissances que nous sommes en droit d'attendre d'une architecture bouleversante - le dépaysement quotidien - sont sacrifiés au vide-ordures que l'on utilisera jamais pour la Bible réglementaire, déjà en place dans les

\footnotetext{
${ }^{6}$ « Construction de taudis » in Guy Debord présente Potlatch (1954-1957), Paris, Gallimard, 1996, p. 25-26.
} 


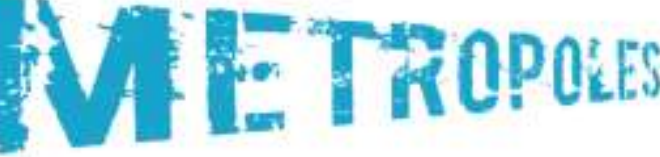

\section{$2008, n^{\circ} 4$}

hôtels des USA ${ }^{7}$. A l'évidence, le groupe de Guy Debord n'a pas vraiment perçu la dimension poétique et polémique des textes de Le Corbusier. Il a néanmoins compris que c'est encore au nom de la morale chrétienne et des impératifs de la société de consommation que l'architecte prétend reconstruire un homme nouveau. C'est pourquoi il dénoncera vite la logique trahie du fonctionnalisme: quoi qu'en disent Le Corbusier et, à sa suite, les architectes rationalistes, l'habitat construit selon le modèle de l'Existenzminimum ne sera jamais riche en surprises et en expériences inédites ${ }^{8}$. Doter cet habitat des éléments du confort moderne ne change rien à l'affaire. Pour les lettristes, le confort est déjà le signe de cette société du spectacle que Debord dénoncera en 1967. Ce n'est pas simplement parce que le confort témoigne de ce nouveau rapport social entre les personnes, tout entier médiatisé par les images de la publicité et qui fait de la possession des objets la condition du bien-être domestique. C'est surtout parce que les technologies du confort suppriment toutes les médiations grâce auxquelles l'espace devient visible et tangible. Contrairement aux effets d'annonce, les technologies domestiques ne recherchent nullement la suppression de la gêne, condition du confort véritable, mais uniquement celle de l'effort. Toutes ensembles, elles contribuent à maintenir l'individu dans un état de passivité où, progressivement, après que ces gestes ont perdu toute circonspection, son corps cesse d'être un instrument d'évaluation de l'espace. A terme l'individu se trouve aliéné à son environnement sans possibilité de s'en affranchir. Pour les futurs situationnistes, il importe bien plutôt de retrouver une intelligence de l'espace domestique en proposant notamment un habitat modulable au sein duquel le corps serait en interaction constante avec son environnement, se découvrirait dans son mouvement, inaugurerait des pratiques nouvelles.

Le même problème se retrouve à l'échelle de la ville : le citadin ne peut pas se libérer de son environnement immédiat, il ne peut pas se soustraire au règne de la quotidienneté parce que les bâtiments dans lesquels il habite, par leur monumentalité, déchirent le tissu urbain et s'imposent dans la ville, ou à ses marges, comme des îlots de silence. Là encore l'Internationale lettriste accuse Le Corbusier de poursuivre l'entreprise de domestication et de contrôle commencée par Haussmann : «Aujourd'hui, la prison devient l'habitation-modèle, et

\footnotetext{
${ }^{7}$ «Les gratte-ciel par la racine » in Guy Debord..., p. 38.

${ }^{8}$ Voir sur ce point les analyses de Jean-Louis Violeau, Situations construites, Paris, Sens et Tonka, 1998,93 p.
} 


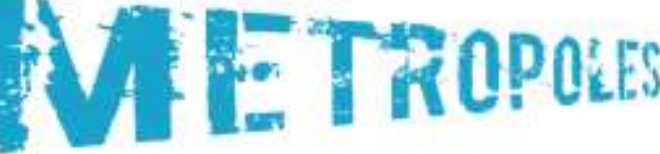

\section{$2008, n^{\circ} 4$}

la morale chrétienne triomphe sans réplique, quand on s'avise que Le Corbusier ambitionne de supprimer la rue. Car il s'en flatte. Voilà le programme : la vie définitivement partagée en îlots fermés, en sociétés surveillées ; la fin des chances d'insurrection et de rencontres ; la résignation automatique $»$. Pour 1'Internationale lettriste comme, plus tard, pour les situationnistes, l'air de la ville ne rend plus libre, ainsi que le proclamait encore Max Weber mais il sent l'ordre. L'urbanisation intensive des modernes n'est pas au service de la ville et de ses habitants. Elle représente au contraire la mise en place d'un dispositif d'isolement, d'exclusion et de réclusion des citadins; elle contribue à l'établissement d'un ordre dans lequel le désir n'a pas sa place, sinon comme désir d'objets. Debord nous rappelle avec force que la réorganisation du tissu urbain se polarise désormais autour de ces nouveaux temples de la consommation que sont les hypermarchés, en rationalisant des parcours, des horaires et des pratiques. La disciplinarisation de l'espace commue ainsi les velléités de contestation en pratiques de consommation.

Pour les situationnistes, la critique de l'urbanisme moderne recouvre donc l'injonction énoncée en 1946 par Lefebvre : celle de la réinvention du quotidien. Face à une culture de masse de plus en plus asphyxiante et un environnement déformé par le culte de la marchandise, il importe de faire renaître le désir au cœur de la ville, d'y introduire des vertiges et des troubles insoupçonnés, d'y inventer des formes de vie inédites et de lui offrir l'événementialité dont elle est aujourd'hui dépourvue. Reste néanmoins à déterminer la façon dont s'opérera cette révolution urbaine.

\section{Le grand jeu à venir}

C'est dans le modèle du joueur, de l'Homo ludens, tel que l'a décrit en 1938 l'historien hollandais Johan Huizinga que Debord et Lefebvre vont respectivement trouver une réponse. Dans son essai sur la fonction sociale du jeu Huizinga voit dans la pratique ludique « une action libre, sentie comme fictive et située en dehors de la vie courante, capable néanmoins d'absorber totalement le joueur; une action dénuée de tout intérêt matériel et de toute

\footnotetext{
${ }^{9}$ « Les gratte-ciel par la racine » in Guy Debord..., p. 38.
} 


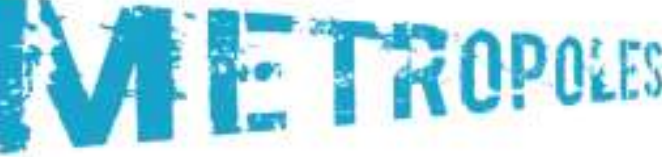

\section{$2008, n^{\circ} 4$}

utilité $»^{10}$. Ce qui intéresse ici Debord comme Lefebvre, c'est bien sûr le fait que le jeu soit à la fois une source majeure de création culturelle et le paradigme de toute attitude antiobjectivante. Lefebvre dit ainsi : «Le jeu, à notre avis, est multiforme et multiple. Loisir et jeu ne se recouvrent pas exactement. Ne serait-ce pas le jeu qui parachève et couronne la sociabilité ? [...] Le jeu ne correspond à aucun besoin élémentaire, encore qu'il les présuppose tous. Il correspond à des désirs affinés et différenciés, selon les individus et les groupes, désirs qui tuent vite la monotonie et l'absence de possibilités ${ }^{11}$. Le jeu n'est donc pas simplement l'expression d'un désir qui met le monde en chantier, il est aussi ce qui en réinvente constamment - mais toujours provisoirement - les règles. Or, c'est précisément ce qui manque à la ville. Les règles que l'urbanisme moderne propose manquent tellement de plasticité qu'il n'est même plus possible de se perdre dans la ville, c'est-à-dire d'être pris à son jeu. Car l'art de se perdre en ville comme on se perd dans un labyrinthe, cet art dont Benjamin disait qu'il demande toute une éducation, tombe lui-même en désuétude. La ville est-elle encore seulement la réalisation de ce rêve ancien de l'humanité qu'est le labyrinthe et auquel le flâneur, sans même y songer, se consacrait pleinement ? Rien n'est moins sûr et Debord nous rappelle bien, non sans amertume, qu'« on peut découvrir d'un seul coup d'œil, l'ordonnance cartésienne du prétendu « labyrinthe » du Jardin des Plantes et l'inscription qui l'annonce : LES JEUX SONT INTERDITS DANS LE LABYRINTHE. On ne saurait trouver un résumé plus clair de l'esprit de toute une civilisation. Celle-là même que nous finirons par abattre $»^{12}$.

Pour défaire «l'ordonnance cartésienne » des villes, les situationnistes vont promouvoir de multiples stratégies ludiques ${ }^{13}$ dont la plus subversive est sans conteste celle de la dérive. La pratique n'a rien d'inédit. On pense immédiatement à la flânerie baudelairienne et aux errances surréalistes, deux modes d'expérimentation qui partagent la même représentation de la ville comme réservoirs de virtualités, avec ses pôles d'attraction et ses brèches dans la

\footnotetext{
${ }^{10}$ Huizinga, Johan, Homo Ludens, Essai sur la fonction sociale du jeu, Paris, Tel Gallimard, 1988, p. 12.

${ }^{11}$ H. Lefebvre, «Utopie expérimentale : Pour un nouvel urbanisme », Revue Française de Sociologie, $n^{\circ} 3$, 1961, p. 197.

12 «Ariane en chômage » in Guy Debord..., p. 71.

${ }^{13}$ Les plus fréquentes sont le détournement d'images et le palindrome. Voir « Le détournement comme négation et comme prélude », I.S. $\mathrm{n}^{\circ} 3$.
} 


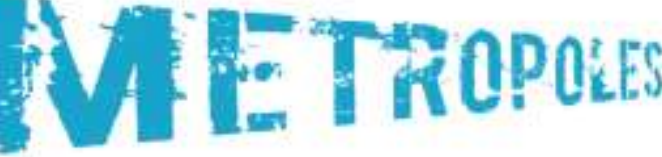

\section{$2008, n^{\circ} 4$}

banalité des espaces balisés ${ }^{14}$. Néanmoins, pour Debord, la dérive n'est pas seulement un mode de comportement expérimental mais possède avant tout une vertu heuristique. En cela, elle n'est pas tant un moyen pour s'évader du quotidien qu'un instrument pour le réinventer. Dans sa Théorie de la dérive de 1958, il en donne la définition suivante : «technique du passage hâtif à travers des ambiances variées. Le concept de dérive est indissolublement lié à la reconnaissance d'effets de nature psychogéographique, et à l'affirmation d'un comportement ludique-constructif, ce qui l'oppose en tous points aux notions classiques de voyage et de promenade. Une ou plusieurs personnes se livrant à la dérive renoncent, pour une durée plus ou moins longue, aux raisons de se déplacer et d'agir qu'elles se connaissent généralement, aux relations, aux travaux et aux loisirs qui leur sont propres, pour se laisser aller aux sollicitations du terrain et des rencontres qui y correspondent $»^{15}$. On retrouve ici les caractéristiques du jeu évoquées par Huizinga (la rupture avec le quotidien, l'anti-utilitarisme, la relation de groupe, etc.) mais on trouve surtout l'idée d'un mode d'exploration de la ville par le jeu.

A la pratique de la dérive comme expérience vécue s'ajoute, en effet, une phase d'analyse visant à repérer les articulations psychogéographiques de la ville, c'est-à-dire «les effets précis du milieu géographique, consciemment aménagé ou non, agissant directement sur le comportement affectif des individus $»^{16}$. La pratique de la dérive est en fait indissociable d'une appréhension psychogéographique de l'espace urbain : si la déambulation implique le laisser-aller, la spontanéité, le refus de parcours assurant l'expression totale des désirs, ces derniers, en retour, doivent contribuer à la localisation de zones d'ambiance dont les potentialités doivent être minutieusement évaluées. La dérive qui assume la dimension ludique de l'exploration urbaine se double donc du repérage psychogéographique dont la fonction constructive est plus manifeste ${ }^{17}$. C'est pourquoi Debord aura rapidement l'idée de

\footnotetext{
${ }^{14}$ Il est intéressant de noter que, contrairement à bon nombre d'utopies urbaines et architecturales qui n'accordent qu'un rôle subsidiaire aux modalités d'occupation libre de l'espace, les pratiques ludiques de l'espace urbain évoquées par Benjamin, Breton ou Debord se fondent toutes sur une préséance du piéton, sur les pouvoirs de la déambulation, toujours définie comme la vraie capacité du citadin d'interagir avec son environnement proche et, par suite, de le redéfinir.

15 «Théorie de la dérive », I.S. n 2, in Internationale situationniste (1958-1969), Paris, Fayard, 1997, p. 51.

16 «Essai de description psychogéographique des Halles », ibid., p. 45.

${ }^{17}$ Ce point permet de mieux comprendre le caractère formel qu'a recouvert la pratique de la dérive chez les situationnistes : elle dure généralement une journée, les participants sont divisés en groupes, munis de talkies-
} 


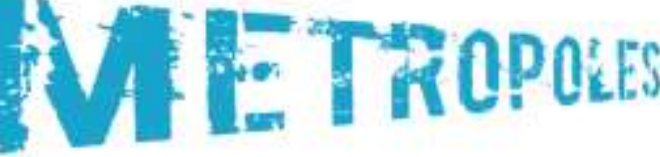

\section{$2008, n^{\circ} 4$}

donner une expression graphique à l'expérience de la dérive. L'objectif sera de détourner des cartes de villes pour les transformer en cartes psychogéographiques qui mettraient au jour la structure cachée des espaces urbains ${ }^{18}$.

La pratique de la dérive mise en place par Debord, son objectivation dans des cartes psychogéographiques vont avoir une influence déterminante sur un des membres de l'Internationale situationniste : Constant Nieuwenhuis. Ancien du groupe Cobra, il rejoint les situationnistes en 1957, partageant avec eux la même aversion pour le fonctionnalisme. Il récuse tout particulièrement l'utilitarisme affiché du mouvement moderne. Selon lui, les quatre fonctions sur lesquelles se fonde l'organisation « rationnelle » de la ville désignent un type d'individu qui ne vaut que par sa capacité à produire de la richesse : habiter, travailler, circuler, se recréer ne sont là que des moyens pour rendre l'homo faber plus productif. Rien d'étonnant alors à ce que l'espace public, libre lieu de rencontres et d'échanges, disparaisse au profit d'un quadrillage urbain où chacun se voit désigner sa place. Constant est convaincu que la peinture et la sculpture représentent des moyens efficaces pour lutter contre cette disciplinarisation de l'espace s'ils contribuent ensembles à la création d'une cité nouvelle. Il rédige avec Debord en 1958 la Déclaration d'Amsterdam qui entend proposer un nouveau mode d'organisation de l'espace, l'urbanisme unitaire. Celui-ci y est défini comme «la théorie de l'emploi d'ensemble des arts et techniques concourant à la construction intégrale d'un milieu en liaison dynamique avec des expériences de comportement ${ }^{19}$. En langage clair, il s'agit de refonder le cadre social en utilisant tous les arts et les technologies disponibles, de créer des ambiances inédites permettant la construction de situations, c'est-àdire des moments de vies à la fois singuliers et éphémères.

En fait, Constant s'est mis depuis longtemps au travail. Il a entrepris un certain nombre d'expérimentations géométriques qui se trouveront rassemblées au Stedelijk Muséum d'Amsterdam en 1959, dans une exposition censée montrer la voie de ce que sera l'urbanisme

\footnotetext{
walkies, échangeant systématiquement leurs impressions, etc. La codification des modalités d'expérience est considérée ici comme la condition nécessaire à l'établissement d'un nouveau savoir urbain.

${ }^{18}$ C'est à partir de 1956 que Debord et Asger Jorn, ex-membre du groupe Cobra, vont produire plusieurs cartes de ce genre : Le guide psychogéographique de Paris, Le discours sur les passions de l'amour, qui reprend le titre d'un ouvrage de Pascal et surtout The Naked City, inspirée de la Carte du tendre de Madame de Scudéry.

${ }^{19}$ « Définitions », Ibid. p. 13.
} 


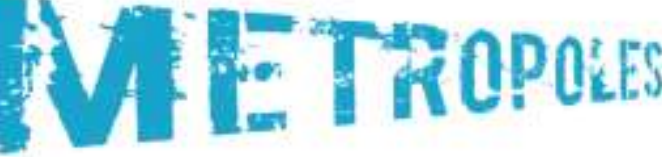

\section{$2008, n^{\circ} 4$}

unitaire. Les maquettes qu'on peut y voir marquent « le passage, à l'intérieur de la production artistique moderne, de l'objet marchandise suffisant à lui-même et dont la fonction est d'être simplement regardé, à l'objet-projet dont la valorisation plus complexe en appelle à une action à mener, action d'un type supérieur concernant la totalité de la vie ». L'intérêt de la démarche ne réside pas seulement dans la tentative d'un dépassement de l'art, au delà de l'économie marchande, mais avant tout dans une pensée de l'espace avec lequel l'individu n'entretient plus un rapport instrumental ou consumériste. Ce projet d'un espace autre, susceptible d'opérer la réinvention du quotidien, va permettre à Constant de jeter les bases d'une cité future, New Babylon, destinée à une société post-révolutionnaire. Partant du postulat selon lequel le développement du machinisme et l'accélération de la production automatisée libérera l'individu de l'aliénation et lui restituera sa liberté créatrice, Constant imagine une ville consacrée exclusivement aux loisirs. L'avènement de l'homo ludens commuera l'espace urbain en gigantesque terrain de jeu, offrant des possibilités de rencontres, d'expériences et de surprises sans cesse renouvelées. Ce renouvellement sera assuré grâce à un dispositif de désordre et de désorientation systématiques. Là encore on retrouve le modèle du labyrinthe. Constant dit à ce propos que «l'espace de New Babylon a toutes les caractéristiques d'un espace labyrinthique à l'intérieur duquel les mouvements ne subissent plus la contrainte de quelque organisation spatiale ou temporelle $»^{20}$. La mobilité des citadins ne rencontrant plus d'obstacles devient une dérive continue. Mais cette transformation du mode d'occupation de l'espace n'est possible que si le labyrinthe est lui-même changeant : «la libération du comportement exige un espace social, labyrinthique mais en même temps continuellement modifiable. Il n'y aura plus de centre à atteindre mais un nombre infini de centres qui se déplacent. Il ne pourra plus être question de s'égarer dans le sens de «se perdre » mais dans le sens plus positif de «trouver des chemins inconnus $»^{21}$. Le modèle du labyrinthe dynamique introduit donc au sein de l'espace social la nécessité de connections différentielles des différents secteurs urbains, se déployant sans programme en de multiples directions. A terme, ce qui est recherché c'est une intensification radicale de l'espace et du temps, pleinement occupé, continûment éprouvé.

\footnotetext{
${ }^{20}$ Constant, New Babylon, Art et utopie, textes situationnistes, édition établie et présentée par JeanClarence Lambert, Paris, Cercle d'Art, 1997, p. 90.

${ }^{21}$ Constant, Ibid. p. 123.
} 


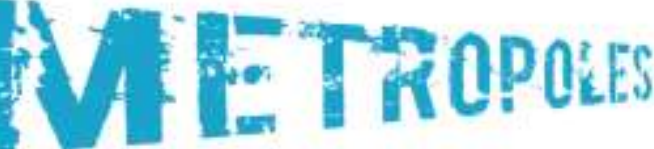

\section{$2008, n^{\circ} 4$}

\section{A la dérive?}

De cette collaboration continue entre Debord et Constant depuis 1956 on aurait pu s'attendre à ce que l'urbanisme unitaire et la dérive présentés comme moyens de connaissances inédits de la ville, contribuent à la réalisation d'un autre espace de vie, tel que le projet de $\mathrm{New}$ Babylon semblait le préfigurer. Debord n'avait-il pas dit que « les hasards et les pouvoirs de l'urbanisme, que nous nous contentons actuellement d'utiliser, nous ne cesserons pas de nous fixer pour but de participer, dans la plus large mesure possible, à leur construction réelle $»^{22}$. Mais qu'entendait-il, au juste, par construction réelle? S'agissait-il vraiment, comme le faisait Constant, de concevoir une architecture et un urbanisme nouveau ? Non, et Debord, sur ce point, va clarifier sa position en 1959: «l'urbanisme unitaire n'est pas une doctrine d'urbanisme mais une critique de l'urbanisme. [...] Aucune discipline séparée ne peut être acceptée en elle-même, nous allons vers une création globale de l'existence ${ }^{23}$. Pour Debord, contrairement à Constant, l'urbanisme unitaire est plus un instrument de contestation visant à renverser l'organisation dominante de la vie qu'un programme architectural virtuel. Il précisera plus tard sa pensée dans la thèse 179 de La société du spectacle en ajoutant que «la plus grande idée révolutionnaire à propos de l'urbanisme n'est pas elle-même urbanistique [...]. C'est la décision de reconstruire intégralement le territoire selon les besoins du pouvoir des Conseils de travailleurs $»^{24}$. Constant, lui, voit les choses différemment. Les temps ne sont pas encore mûrs pour une transformation révolutionnaire de la société mais, en attendant, les créateurs ne doivent nullement garder le silence. Ils restent des concepteurs et, faute de mieux, des « semi-joueurs », les interprètes de l'homme à venir, de l'homo ludens. Plus que jamais, il est temps d'imaginer une « autre ville pour une autre vie $»^{25}$.

On voit ici comment, au début des années soixante, une ligne de partage s'est dessinée entre Debord et Constant. Ce dernier finira par rompre avec les situationnistes et continuera seul la poursuite de son utopie urbaine. Quant au mouvement situationniste, on peut constater qu'il s'est lui aussi éloigné de ses préoccupations initiales. Est-il parti à la dérive ? En un sens, les

\footnotetext{
22 «L'architecture et le jeu » in Guy Debord..., p. 158.

${ }^{23}$ «L'urbanisme unitaire à la fin des années 50 », I.S. n 3, in Internationale situationniste..., p. 80.

${ }^{24}$ Debord, Guy, La société du spectacle, Paris, Gallimard, 1992, p. 136-137.

${ }^{25}$ Titre d'un texte de Constant publié en 1959 dans le bulletin $n^{\circ} 3$ de l'I.S.
} 


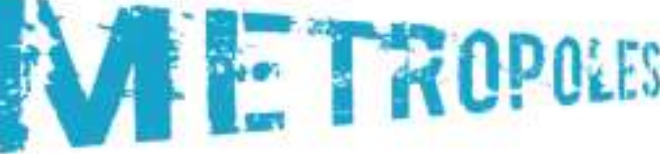

\section{$2008, n^{\circ} 4$}

pratiques ludiques des situationnistes se prolongeront et s'éprouveront plus radicalement dans les événements de mai 68, notamment avec la construction de barricades. Néanmoins, concernant les perspectives de l'urbanisme unitaire, on ne peut s'empêcher de penser qu'en rompant avec toute pratique effective de l'architecture et de l'urbanisme les situationnistes n'ont jamais pu expérimenter les modalités d'une réinvention du quotidien. Il est toujours possible d'admettre qu'ils ont été des constructeurs de situations, d'ambiances transitoires. Mais comment celles-ci auraient-elles pu subvertir l'ordre des formes urbaines dominantes dans la mesure où, d'une part, aucune d'elles n'avaient aucun caractère durable et, d'autre part, elles furent elles-mêmes discréditées au nom d'une théorie générale de la révolution. Dans le dernier tome de sa Critique de la vie quotidienne, Lefebvre portera un constat désabusé sur le mouvement de Guy Debord : «La théorie de la révolution dans le quotidien devait avoir des répercussions imprévues. La connaissance critique allait engendrer l'hypercriticisme, à la limite la pure et simple négation abstraite de l'existant, le refus du « réel » traité comme un théâtre d'ombre. [...] Sur la voie de l'hypercriticisme, l'intelligentsia gauchiste a démoli toute les valeurs, avec d'excellentes raisons, mais en détruisant les raisons de vivre. Pour employer une vieille métaphore, elle a scié la branche sur laquelle elle était assise. Conduite suicidaire $»^{26}$. De façon singulière, le destin des situationnistes rappelle celui des surréalistes dont ils entendaient pourtant se démarquer radicalement : si «l'amère victoire du Surréalisme » fut sans doute d'avoir plus contribué à changer le paysage littéraire que l'ordre social dominant, l'écueil des situationnistes est peut-être de n'avoir pas œuvré du tout. En récusant la séparation qu'implique toute pratique artistique, en refusant de produire des œuvres qui puissent, à terme, avoir leur vocation propre, l'Internationale situationniste s'est peu à peu vouée à l'impuissance, refermant sur elle-même les espoirs d'une esthétique de l'organisation révolutionnaire qui, pourtant, voulait tout à la fois changer la ville, changer la vie.

\footnotetext{
${ }^{26}$ H. Lefebvre, La critique de la vie quotidienne, t. 3, Paris, L’arche, 1981, p. 35-36.
} 